\title{
Revisiting Ramaria species: the Coral Fungi as Food and Pharmaceuticals
}

\author{
Sandipta Ghosh ${ }^{1, \dagger}$ (i) , Tribeni Chatterjee ${ }^{1,+}$ (i) , Krishnendu Acharya $1, *$ (D) \\ 1 Molecular and Applied Mycology and Plant Pathology Laboratory, Department of Botany, University of Calcutta, \\ Kolkata-700019, India \\ $\dagger \quad$ These authors contributed equally to this paper \\ * Correspondence: krish_paper@yahoo.com;
}

Scopus Author ID 42761171800

Received: 8.10.2020; Revised: 9.11.2020; Accepted: 12.11.2020; Published: 15.11.2020

\begin{abstract}
With the advancement of the quality of life and the resultant increase in the frequency of lifethreatening diseases, food is being viewed as a source of nutritional and functional benefits. Mushrooms have served as nutritious food from time immemorial along with useful medicinal properties. The genus Ramaria, coral fungi, is a potential group of culinarily acclaimed mushrooms with worldwide distribution. During the past two decades, studies on several species of the genus revealed high medicinal potency. In this review, we particularly aim to update this group's present status with respect to its importance as food and medicine, which will offer a new perception to researchers for its progress from dietary food to functional food.
\end{abstract}

Keywords: bioactive metabolites; functional food; mycomedicine; Ramaria sp.

(C) 2020 by the authors. This article is an open-access article distributed under the terms and conditions of the Creative Commons Attribution (CC BY) license (https://creativecommons.org/licenses/by/4.0/).

\section{Introduction}

Mushrooms are famous as therapeutic food since prehistoric times. A global population of about 140,000 mushroom species has been estimated, of which only $10 \%$ has been recorded well investigated [1]. It is believed that food and medicines share a common point of origin, and mushrooms are one of the most suitable manifestations of this idea [2]. They possess immense potential in bioprospecting based on their colossal roles in the production of ecologically beneficial bioactive metabolites, ethnomedical uses as well as enriched possibilities for genetic, pharmacological, and chemical analysis [3-5].

Ramaria Fr. ex Bonord is one of the most beautiful coralloid fungi with conspicuously colored basidiomata and circuitous branching pattern, bearing similarities with the genus Clavaria, because of which Persoon had described Ramaria botrytis and mistakenly placed it under the genus Clavaria $[6,7]$. This genus contains 336 species, among which many species are distributed worldwide. From the Indian subcontinent, only 51 species have been reported to date [8]. However, the literature on this genus has been limited to its resource investigation and identification. Many species of this genus are ethnically edible such as $R$. versatilis, $R$. aurea, $R$. botrytis, and others. In contrast, a few species possess twofold traits as a consumable and ectomycorrhizal association [9-11].

The aim of this review is to compile recent data on the nutritional and medicinal properties of different species belonging to this genus. 


\section{Proximate composition of Ramaria species}

The proximate composition of mushrooms is defined in terms of six parameters, among which moisture content is one of the essential factors that determine the freshness of tissue. Concerning Ramaria species, moisture content was relatively high in $R$. botrytis and $R$. flava, as reported in the literature $[12,13]$. The proximate composition of Ramaria species is presented in Table 1.

Carbohydrates and crude protein are considered as such significant components that comprise the bulk of mushroom fruiting bodies. According to the table, the sugar content of Ramaria species varies with different regions of collections throughout the globe. The variability of carbohydrates and protein content among different Ramaria sp. seems to be associated with varying strains, substrate composition, and others. Mushrooms are a preferred source of nutrition because of their high-quality carbohydrate and protein and their low-fat content and high content of polyunsaturated fatty acids (PUFA) [14]. From the literature survey, it has been observed that the amount of lipids/fats in Ramaria sp. ranges from $0.14 \%$ $5.67 \%$ on a dry weight basis (Table 1). According to reports, Ramaria sp. contains many unsaturated fatty acids, especially MUFA and PUFA.

In contrast, linoleic acid (8.28\%) and eicosadienoic acid (4.20\%) were the most abundant PUFA in $R$. botrytis[15]. Thus, cumulatively, Ramaria species have significant nutritional attributes and can be listed in nutrition-rich food items. However, information about the digestibility and bioavailability of these mushroom nutrients has lacked a further in-depth study of these species.

Table 1. Nutritive property of different species of Ramaria.

\begin{tabular}{|c|c|c|c|c|c|c|c|c|c|c|}
\hline $\begin{array}{l}\text { Nutritional } \\
\text { parameters } \\
(\%)\end{array}$ & $\begin{array}{l}R . \\
\text { largentii }\end{array}$ & $\begin{array}{l}R . \\
\text { patagonica }\end{array}$ & $\begin{array}{l}R \text {. } \\
\text { rubripermanens }\end{array}$ & $\begin{array}{l}R . \\
\text { brevispora }\end{array}$ & $\begin{array}{l}R . \\
\text { flava }\end{array}$ & $\begin{array}{l}R . \\
\text { flavescens }\end{array}$ & $\begin{array}{l}R . \\
\text { aurea }\end{array}$ & $\begin{array}{l}R . \\
\text { stricta }\end{array}$ & \multicolumn{2}{|c|}{$R$. botrytis } \\
\hline Carbohydrate & 58.87 & 69.43 & 48.82 & ND & 46.54 & 45.26 & 43.82 & 40.50 & 50.25 & 5.12 \\
\hline Protein & 28.8 & 19.68 & 16.32 & 24.1 & 15.8 & 14.60 & 13.30 & 10.81 & 21.65 & 4.08 \\
\hline Fat & 5.67 & 2.51 & 1.49 & 1.30 & 1.26 & 1.18 & 0.91 & 0.37 & 0.22 & 0.14 \\
\hline Dietary fibres & ND & ND & 1.06 & 8.8 & 1.02 & 0.90 & 0.82 & 0.28 & 1.33 & ND \\
\hline Moisture & ND & ND & ND & ND & 93.3 & $\mathrm{ND}$ & ND & ND & 89.77 & 89.77 \\
\hline Reference & [53] & [21] & [15] & [54] & [15] & {$[15]$} & [15] & [15] & {$[15]$} & {$[12]$} \\
\hline
\end{tabular}

ND: not detected

\section{Bioactive secondary metabolites}

Mushrooms are often considered a storehouse of bioactive metabolites that show immense potential as promising therapeutic agents [16]. These include phenolic compounds, terpenoids, sterols, and others, which play a vital role as scavengers of free radicals and thus stabilize lipid oxidation. Several studies have been conducted to ascertain the amount of bioactive secondary metabolites in different species of Ramaria.

The most extensively studied species is $R$. flava. The ethanolic extract of this mushroom contained higher phenolic content than methanolic extract [17-19]. Moreover, highperformance liquid chromatogram analysis of an ethanolic extract of $R$. flava from Mongolia revealed the presence of quercetin, chrysin, and pinocembrin. The hydromethanolic extract of $R$. largentii revealed the presence of protocatechuic and vanillic acids [20]. Furthermore, the methanolic extract of $R$. patagonica [21], $R$. formosa [22], and $R$. subalpina [23] was reported to contain phenolic compounds, including gallic acid, p-hydroxybenzoic acid, p-coumaric acid. Other than alcoholic extract, Khatua et al. [24] determined phenolic constituents of a heatstable polyphenol-rich extract of $R$. aurea in which caffeic acid was found as the most abundant 
phenolics followed by cinnamic acid and gallic acid. On the other hand, Ramaria sp. was also reported to contain carotenoids, ascorbic acid, anthocyanidins, and tocopherols [15]. $\beta$ tocopherol was found to be present in a higher amount than $\alpha$ tocopherol, the active form of Vitamin E.

\section{Ramaria species and their prospective medicinal properties}

\subsection{Antioxidant activity.}

A wide variety of reports regarding antioxidative properties of different Ramaria sp. have been published, which revealed the potentiality of these fungi as an effective antioxidant. The majority of reports showed radical scavenging activity of extracts tested in in-vitro systems; only two reports described in-vivo experiments (Table 2). R. flava [17-19] and $R$. botrytis $[12,15,25]$ were the most extensively studied species of this group. The methanolic and ethanolic extracts of these two species were able to scavenge free radicals, which seem to occur because of synergistic effects of different phenolic compounds extracted through experiments. Besides scavenging free radicals, Ramaria species also showed positive results in lipid peroxidation inhibition assay, iron-chelating assay, reducing power assay, and 15lipoxygenase inhibition (Table 2). The methanolic extract $R$. botrytis was also tested to determine its antioxidant potential in both in vitro and in vivo system. The extract was found to reduce superoxide dismutase (SOD), catalase, and glutathione peroxidase levels and inhibit lipid peroxide content in the liver of benzo- $\alpha$ pyrene treated mice [26].

Table 2. Antioxidant activities of different species of Ramaria.

\begin{tabular}{|c|c|c|c|c|c|}
\hline Species & $\begin{array}{l}\text { Collection } \\
\text { place }\end{array}$ & Methods used & Extracts used & $\begin{array}{l}\text { Activity (EC } \mathrm{E}_{50} / \% \text { of } \\
\text { inhibition) }\end{array}$ & References \\
\hline \multirow[t]{4}{*}{ R. flava } & \multirow[t]{4}{*}{ Turkey } & $\begin{array}{l}\beta \text {-carotene linoleic acid } \\
\text { assay }\end{array}$ & \multirow[t]{4}{*}{ Methanol } & $95.02 \%$ at $20 \mathrm{mg} / \mathrm{mL}$ & \multirow[t]{4}{*}{ [17] } \\
\hline & & DPPH scavenging assay & & $94.78 \%$ at $12 \mathrm{mg} / \mathrm{mL}$ & \\
\hline & & Reducing power & & 1.91 at $20 \mathrm{mg} / \mathrm{mL}$ & \\
\hline & & Metal chelating effect & & 96.75 at $2 \mathrm{mg} / \mathrm{mL}$ & \\
\hline R. formosa & India & $\begin{array}{l}\text { DPPH radical scavenging } \\
\text { activity }\end{array}$ & Methanol & $\mathrm{EC}_{50} 5.8 \mathrm{mg} / \mathrm{ml}$ & {$[22]$} \\
\hline \multirow[t]{12}{*}{ R. flava } & \multirow[t]{2}{*}{ Turkey } & DPPH scavenging assay & \multirow[t]{2}{*}{ Ethanol } & $\mathrm{EC}_{50} 276 \mu \mathrm{g} / \mathrm{mL}$ & \multirow[t]{2}{*}[18]{} \\
\hline & & $\begin{array}{l}\beta \text {-carotene linoleic acid } \\
\text { assay }\end{array}$ & & $94.7 \%$ at $160 \mu \mathrm{g} / \mathrm{mL}$ & \\
\hline & \multirow[t]{10}{*}{ China } & \multirow{5}{*}{$\begin{array}{l}\text { DPPH radical-scavenging } \\
\text { activity }\end{array}$} & Ethanol & $\mathrm{EC}_{50} 34.41 \mu \mathrm{g} / \mathrm{mL}$ & \multirow[t]{10}{*}{ [19] } \\
\hline & & & Petroleum ether & $\mathrm{EC}_{50} 42.29 \mu \mathrm{g} / \mathrm{mL}$ & \\
\hline & & & Ethyl acetate & $\mathrm{EC}_{50} 20.37 \mu \mathrm{g} / \mathrm{mL}$ & \\
\hline & & & n-butanol & $\mathrm{EC}_{50} 46.80 \mu \mathrm{g} / \mathrm{mL}$ & \\
\hline & & & Water & $\mathrm{EC}_{50} 5.86 \mu \mathrm{g} / \mathrm{mL}$ & \\
\hline & & \multirow{5}{*}{$\begin{array}{l}\text { Hydroxyl radical- } \\
\text { scavenging activity assay }\end{array}$} & Ethanol & $\mathrm{EC}_{50} 41.46 \mu \mathrm{g} / \mathrm{mL}$ & \\
\hline & & & Petroleum ether & $\mathrm{EC}_{50} 102.43 \mu \mathrm{g} / \mathrm{mL}$ & \\
\hline & & & Ethyl acetate & $\mathrm{EC}_{50} 32.41 \mu \mathrm{g} / \mathrm{mL}$ & \\
\hline & & & n-butanol & $\mathrm{EC}_{50} 116.55 \mu \mathrm{g} / \mathrm{mL}$ & \\
\hline & & & Water & $\mathrm{EC}_{50} 18.08 \mu \mathrm{g} / \mathrm{mL}$ & \\
\hline \multirow[t]{9}{*}{$\begin{array}{ll}R . & \text { botrytis } \\
\text { (Pers.) } & \end{array}$} & \multirow[t]{9}{*}{ China } & \multirow[t]{4}{*}{$\begin{array}{l}\text { DPPH radical-scavenging } \\
\text { activity }\end{array}$} & $\begin{array}{l}\text { Purified } \\
\text { polysaccharide } \\
\text { (RBP) } \\
\text { RBP1 }\end{array}$ & $44.33 \%$ at $1.4 \mathrm{mg} / \mathrm{mL}$ & \multirow[t]{9}{*}{ [55] } \\
\hline & & & RBP2 & $14.67 \%$ at $1.4 \mathrm{mg} / \mathrm{mL}$ & \\
\hline & & & RBP3 & $74.01 \%$ at $1.4 \mathrm{mg} / \mathrm{mL}$ & \\
\hline & & & RBP4 & $82.67 \%$ at $1.4 \mathrm{mg} / \mathrm{mL}$ & \\
\hline & & \multirow{4}{*}{$\begin{array}{l}\text { Hydroxyl radical } \\
\text { scavenging activity }\end{array}$} & RBP1 & $\sim 19 \%$ at $1.2 \mathrm{mg} / \mathrm{mL}$ & \\
\hline & & & RBP2 & $\sim 50 \%$ at $1.2 \mathrm{mg} / \mathrm{mL}$ & \\
\hline & & & RBP3 & $90 \%$ at $1.2 \mathrm{mg} / \mathrm{mL}$ & \\
\hline & & & RBP4 & $\sim 70 \%$ at $1.2 \mathrm{mg} / \mathrm{mL}$ & \\
\hline & & Reducing power & RPB1 & $\begin{array}{l}0.2 \text { absorbance at } 1.5 \\
\mathrm{mg} / \mathrm{mL}\end{array}$ & \\
\hline
\end{tabular}


https://doi.org/10.33263/BRIAC113.1079010800

\begin{tabular}{|c|c|c|c|c|c|}
\hline Species & $\begin{array}{l}\text { Collection } \\
\text { place }\end{array}$ & Methods used & Extracts used & $\begin{array}{l}\text { Activity (EC50/\% of } \\
\text { inhibition) }\end{array}$ & References \\
\hline & & & RBP2 & $\begin{array}{l}0.8 \text { absorbance at } 1.5 \\
\mathrm{mg} / \mathrm{mL}\end{array}$ & \\
\hline & & & RBP3 & $\begin{array}{l}0.4 \text { absorbance at } 1.5 \\
\mathrm{mg} / \mathrm{mL}\end{array}$ & \\
\hline & & & RBP4 & $\begin{array}{l}0.56 \text { absorbance at } 1.5 \\
\mathrm{mg} / \mathrm{mL}\end{array}$ & \\
\hline \multirow[t]{4}{*}{ R. botrytis } & \multirow[t]{4}{*}{ Portugal } & DPPH scavenging activity & \multirow[t]{4}{*}{ Methanolic extract } & $\mathrm{EC}_{50} 0.66 \mathrm{mg} / \mathrm{mL}$ & \multirow[t]{4}{*}{ [12] } \\
\hline & & Reducing power & & $\mathrm{EC}_{50} 0.68 \mathrm{mg} / \mathrm{mL}$ & \\
\hline & & $\begin{array}{ll}\text {-carotene } & \text { bleaching } \\
\text { inhibition } & \\
\end{array}$ & & $\mathrm{EC}_{50} 0.67 \mathrm{mg} / \mathrm{mL}$ & \\
\hline & & $\begin{array}{ll}\begin{array}{l}\text { Lipid } \\
\text { inhibition }\end{array} & \text { peroxidation } \\
\end{array}$ & & $\mathrm{EC}_{50} 1.01 \mathrm{mg} / \mathrm{mL}$ & \\
\hline \multirow[t]{4}{*}{ R. aurea } & \multirow[t]{4}{*}{ India } & DPPH scavenging activity & \multirow{4}{*}{$\begin{array}{l}\text { Polyphenol rich } \\
\text { ethanolic extract }\end{array}$} & $\mathrm{EC}_{50} 0.283 \mathrm{mg} / \mathrm{mL}$ & \multirow[t]{4}{*}[24]{} \\
\hline & & $\begin{array}{l}\text { Superoxide scavenging } \\
\text { activity }\end{array}$ & & $\mathrm{EC}_{50} 0.384 \mathrm{mg} / \mathrm{ml}$ & \\
\hline & & Chelating ability & & $\mathrm{EC}_{50} 0.95 \mathrm{mg} / \mathrm{ml}$ & \\
\hline & & Reducing power & & $\mathrm{EC}_{50} 1.025 \mathrm{mg} / \mathrm{ml}$ & \\
\hline \multirow[t]{4}{*}{ R. patagonica } & \multirow[t]{4}{*}{ Argentina } & DPPH scavenging activity & \multirow[t]{4}{*}{ Methanol } & $\mathrm{EC}_{50} 770 \mu \mathrm{g} / \mathrm{mL}$ & \multirow[t]{4}{*}[21]{} \\
\hline & & Reducing power & & $\mathrm{EC}_{50} 170 \mu \mathrm{g} / \mathrm{mL}$ & \\
\hline & & $\begin{array}{ll}\beta \text {-carotene bleaching } \\
\text { assay }\end{array}$ & & $\mathrm{EC}_{50} 610 \mu \mathrm{g} / \mathrm{mL}$ & \\
\hline & & TBARS inhibition activity & & $\mathrm{EC}_{50} 60 \mu \mathrm{g} / \mathrm{mL}$ & \\
\hline \multirow[t]{3}{*}{ R. formosa } & \multirow[t]{3}{*}{ Korea } & DPPH scavenging activity & \multirow[t]{3}{*}{ Ethyl acetate } & $\begin{array}{l}117 \mathrm{AsA} / \mathrm{mg} / \mathrm{mL} \text { at } \\
500 \mu \mathrm{g} / \mathrm{mL}\end{array}$ & \multirow[t]{3}{*}[50]{} \\
\hline & & $\begin{array}{l}\text { Peroxyl radical } \\
\text { scavenging activity }\end{array}$ & & 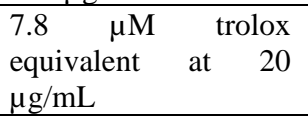 & \\
\hline & & Reducing capacity & & $\begin{array}{lr}36 \% \text { copper } & \text { ion } \\
\text { inhibition at } 20 \\
\mu \mathrm{g} / \mathrm{mL} \\
\text { concentration }\end{array}$ & \\
\hline \multirow[t]{8}{*}{ R. botrytis } & \multirow[t]{8}{*}{ Korea } & \multirow[t]{4}{*}{ DPPH scavenging activity } & Ethanol & $91.4 \%$ & \multirow[t]{8}{*}[25]{} \\
\hline & & & Methanol & $92.1 \%$ & \\
\hline & & & Ethyl acetate & $55.9 \%$ & \\
\hline & & & Acetone & $70 \%$ & \\
\hline & & \multirow[t]{4}{*}{ ABTS scavenging activity } & Ethanol & $50.6 \%$ & \\
\hline & & & Methanol & $87.6 \%$ & \\
\hline & & & Ethyl acetate & $2.8 \%$ & \\
\hline & & & Acetone & $60.8 \%$ & \\
\hline \multirow[t]{5}{*}{ R. botrytis } & \multirow[t]{5}{*}{ India } & $\begin{array}{l}\text { DPPH radical scavenging } \\
\text { activity }\end{array}$ & \multirow[t]{5}{*}{ Ethanol } & $\mathrm{EC}_{50} 680 \mu \mathrm{g} / \mathrm{mL}$ & [15] \\
\hline & & $\begin{array}{l}\text { ABTS radical scavenging } \\
\text { activity }\end{array}$ & & $\mathrm{EC}_{50} 390 \mu \mathrm{g} / \mathrm{mL}$ & \\
\hline & & Ferric ion reducing power & & $\mathrm{EC}_{50} 920 \mu \mathrm{g} / \mathrm{mL}$ & \\
\hline & & Iron chelating ability & & $\mathrm{EC}_{50} 1120 \mu \mathrm{g} / \mathrm{mL}$ & \\
\hline & & $\begin{array}{l}\text { Superoxide anion radical } \\
\text { scavenging activity }\end{array}$ & & $\mathrm{EC}_{50} 869 \mu \mathrm{g} / \mathrm{mL}$ & \\
\hline $\begin{array}{l}R . \\
\text { rubripermanens }\end{array}$ & & $\begin{array}{l}\text { DPPH radical scavenging } \\
\text { activity }\end{array}$ & & $\mathrm{EC}_{50} 760 \mu \mathrm{g} / \mathrm{ml}$ & \\
\hline & & $\begin{array}{l}\text { ABTS } \\
\text { scavenging activity }\end{array}$ & & $\mathrm{EC}_{50} 430 \mu \mathrm{g} / \mathrm{mL}$ & \\
\hline & & Ferric ion reducing power & & $\mathrm{EC}_{50} 1150 \mu \mathrm{g} / \mathrm{mL}$ & \\
\hline & & Iron chelating ability & & $\mathrm{EC}_{50} 1220 \mu \mathrm{g} / \mathrm{mL}$ & \\
\hline & & $\begin{array}{l}\text { Superoxide anion radical } \\
\text { scavenging activity }\end{array}$ & & $\mathrm{EC}_{50} 1120 \mu \mathrm{g} / \mathrm{mL}$ & \\
\hline R. flava & & $\begin{array}{l}\text { DPPH radical scavenging } \\
\text { activity }\end{array}$ & & $\mathrm{EC}_{50} 880 \mu \mathrm{g} / \mathrm{mL}$ & \\
\hline & & $\begin{array}{l}\text { ABTS radical scavenging } \\
\text { activity }\end{array}$ & & $\mathrm{EC}_{50} 490 \mu \mathrm{g} / \mathrm{mL}$ & \\
\hline & & Ferric ion reducing power & & $\mathrm{EC}_{50} 1180 \mu \mathrm{g} / \mathrm{mL}$ & \\
\hline & & Iron chelating ability & & $\mathrm{EC}_{50} 1340 \mu \mathrm{g} / \mathrm{mL}$ & \\
\hline & & $\begin{array}{l}\text { Superoxide anion radical } \\
\text { scavenging activity }\end{array}$ & & $\mathrm{EC}_{50} 1350 \mu \mathrm{g} / \mathrm{mL}$ & \\
\hline R. flavescens & & $\begin{array}{l}\text { DPPH radical scavenging } \\
\text { activity }\end{array}$ & & $\mathrm{EC}_{50} 1520 \mu \mathrm{g} / \mathrm{mL}$ & \\
\hline & & $\begin{array}{l}\text { ABTS radical scavenging } \\
\text { activity }\end{array}$ & & $\mathrm{EC}_{50} 590 \mu \mathrm{g} / \mathrm{mL}$ & \\
\hline
\end{tabular}




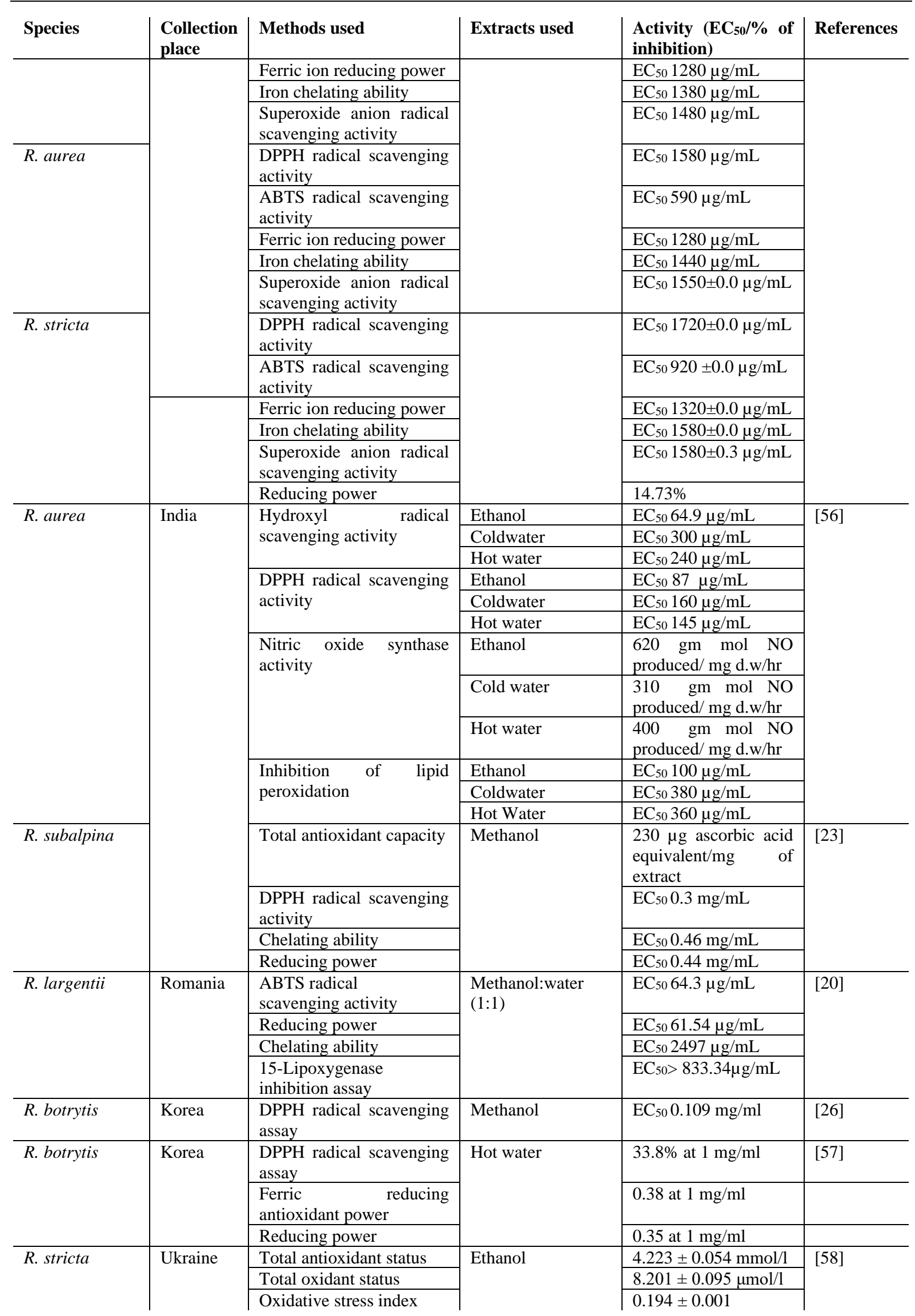

\subsection{Antimicrobial activity.}

Despite the vast diversity of synthetic antibacterial compounds, bacterial resistance to conventional antibiotics has been substantially increasing [27]. Therefore, research of novel antibiotics that are resistant to the current remedy is urgently needed. Since ancient times, 
natural resources have been exploited for humankind. Among them, mushrooms could be an alternative source of new antimicrobials[28-30]. In this context, different Ramaria sp. showed potential antibacterial activity against both gram-negative and gram-positive bacteria (Table 3). Staphylococcus aureus and Pseudomonas aeruginosa were the most sensitive strains to the extract of $R$. botrytis [25], R. zippellii [31]R. aurea [32], R. flava [19] and R. Formosa [22]. However, Bala et al. demonstrated water extracts of Ramaria sp. showed inhibitory action on fungal growth. In contrast, ethanolic extract showed a wider range of antimicrobial activity against the test organisms [33]. Besides, four butenolides (ramariolides A-D) isolated from coral mushroom $R$. cystidiphora showed strong antimicrobial effects against gram-positive bacteria as well as antimycobacterial effects, including Mycobacterium tuberculosis [34]. Later, Lehman et al. investigated the cellular mechanism of ramariolide A in mycobacteria and revealed that ramariolide A binds with a natively folded target protein resulting in amino acid anabolism. However, this fact requires further detailed analysis [35]. Thus it indicates that Ramaria sp. can become a source of antimicrobial leads in the times to come.

Table 3. Antimicrobial activities of different species of Ramaria.

\begin{tabular}{|c|c|c|c|c|c|c|}
\hline \multirow[t]{2}{*}{ Species } & \multirow{2}{*}{$\begin{array}{l}\text { Extracts } \\
\text { used }\end{array}$} & \multirow{2}{*}{$\begin{array}{l}\text { Methods } \\
\text { Used }\end{array}$} & \multicolumn{2}{|c|}{ Bacteria that were inhibited } & \multirow{2}{*}{$\begin{array}{l}\text { Fungi that } \\
\text { were } \\
\text { inhibited }\end{array}$} & \multirow[t]{2}{*}{ References } \\
\hline & & & Gram-positive & Gram-negative & & \\
\hline R. formosa & Methanol & $\begin{array}{l}\text { Inhibition } \\
\text { zone assay }\end{array}$ & S. aureus & P. aeruginosa & $\begin{array}{l}\text { Candida } \\
\text { albicans }\end{array}$ & {$[22]$} \\
\hline R. formosa & $\begin{array}{l}\text { Ethyl } \\
\text { acetate, } \\
\text { methanol, } \\
\text { and water }\end{array}$ & $\begin{array}{l}\text { Percentage of } \\
\text { inhibition }\end{array}$ & $\begin{array}{l}\text { S. aureus, } B . \\
\text { subtilis }\end{array}$ & $\begin{array}{lr}\text { E. coli, } & K . \\
\text { pneumonia, } & P . \\
\text { aeruginosa, } & \\
\text { Proteus vulgaris }\end{array}$ & Not tested & [59] \\
\hline \multirow[t]{2}{*}{ R. zippellii } & Ethanol & \multirow{8}{*}{$\begin{array}{l}\text { 96-well } \\
\text { microplate } \\
\text { bioassay }\end{array}$} & \multirow[t]{6}{*}{ S. aureus } & \multirow[t]{6}{*}{ E. coli } & \multirow[t]{6}{*}{ No activity } & \multirow[t]{6}{*}{ [31] } \\
\hline & Water & & & & & \\
\hline \multirow[t]{2}{*}{ Ramaria sp. 1} & Water & & & & & \\
\hline & Ethanol & & & & & \\
\hline \multirow[t]{2}{*}{ Ramaria sp. 2} & Water & & & & & \\
\hline & Ethanol & & & & & \\
\hline \multirow[t]{2}{*}{ Ramaria sp. } & Water & & \multirow{2}{*}{$\begin{array}{l}\text { Bacillus cereus, } \\
\text { Listeria } \\
\text { monocytogenes }\end{array}$} & \multirow{2}{*}{$\begin{array}{l}P . \quad \text { aeruginosa, } \\
\text { Acinetobacter } \\
\text { baumannii }\end{array}$} & \multirow{2}{*}{$\begin{array}{l}\text { Geotrichum } \\
\text { candidum, S. } \\
\text { cerevisiae }\end{array}$} & \multirow[t]{2}{*}[33]{} \\
\hline & Ethanol & & & & & \\
\hline R. botrytis & Methanol & $\begin{array}{l}\text { Disc diffusion } \\
\text { method }\end{array}$ & No activity & P. vulgaris & No activity & [60] \\
\hline$R$. botrytis & $\begin{array}{l}\text { Methanol- } \\
\text { water }\end{array}$ & $\begin{array}{l}\text { Microdilution } \\
\text { method }\end{array}$ & $\begin{array}{l}\text { Enterococcus } \\
\text { faecalis, } \\
\text { L. } \\
\text { monocytogenes, } \\
\text { Streptococcus } \\
\text { aglactiae, and } \\
\text { Streptococcus } \\
\text { pyrogenes }\end{array}$ & $\begin{array}{l}\text { Pasteurella } \\
\text { multicoda }\end{array}$ & No activity & {$[27]$} \\
\hline R. aurea & Ethanol & $\begin{array}{l}\text { Measurement } \\
\text { of inhibition } \\
\text { zone diameter }\end{array}$ & S. aureus & $\begin{array}{l}P \text {. aeruginosa } \\
\text { E. coli } \\
P . \text { vulgaris }\end{array}$ & C. albicans & {$[32]$} \\
\hline R. flava & Ethanol & $\begin{array}{l}\text { Inhibition } \\
\text { zone assay }\end{array}$ & $\begin{array}{l}\text { S. aureusand } B . \\
\text { subtilis }\end{array}$ & E. coli & $\begin{array}{l}\text { Fusarium } \\
\text { auneaceum, } \\
\text { F. } \\
\text { graminearum } \\
\text { and } \\
\text { Cercosporella } \\
\text { albo- } \\
\text { maculans }\end{array}$ & [19] \\
\hline R. flava & Ethanol & $\begin{array}{l}\text { Agar-well } \\
\text { diffusion } \\
\text { method }\end{array}$ & $\begin{array}{l}\text { Salmonella } \\
\text { enteritidis, } \\
\text { Yersinia } \\
\text { enterecolitica } \\
\text { and } \\
\text { K.pneumoniae }\end{array}$ & $\begin{array}{l}\text { S. aureus, } \\
\text { Micrococcus } \\
\text { luteus, M. flavus, } \\
\text { B. subtilis, B. } \\
\text { cereus }\end{array}$ & No activity & {$[18]$} \\
\hline
\end{tabular}




\begin{tabular}{|c|c|c|c|c|c|c|}
\hline \multirow[t]{2}{*}{ Species } & \multirow{2}{*}{$\begin{array}{l}\text { Extracts } \\
\text { used }\end{array}$} & \multirow{2}{*}{$\begin{array}{l}\text { Methods } \\
\text { Used }\end{array}$} & \multicolumn{2}{|c|}{ Bacteria that were inhibited } & \multirow{2}{*}{$\begin{array}{l}\text { Fungi that } \\
\text { were } \\
\text { inhibited }\end{array}$} & \multirow[t]{2}{*}{ References } \\
\hline & & & Gram-positive & Gram-negative & & \\
\hline$R$. botrytis & $\begin{array}{l}\text { Acetone } \\
\text { Ethanol } \\
\text { Ethyl } \\
\text { Acetate } \\
\text { Methanol }\end{array}$ & $\begin{array}{l}\text { Measurement } \\
\text { of inhibition } \\
\text { zone diameter }\end{array}$ & S. aureus & $\begin{array}{l}P . \text { aeruginosa } \\
\text { E. coli } \\
\text { Enterobacter } \\
\text { cloacae }\end{array}$ & No activity & [25] \\
\hline $\begin{array}{l}R . \text { botrytis } \\
R . \\
\text { rubripermanens } \\
R . \text { flava } \\
R . \text { flavescens } \\
R . \text { aurea } \\
R \text {. stricta }\end{array}$ & Methanol & $\begin{array}{l}\text { Measurement } \\
\text { of minimum } \\
\text { inhibitory } \\
\text { concentrations }\end{array}$ & S. aureus & $\begin{array}{l}\text { E. coli, } \\
\text { K. pneumonia, } \\
\text { Vibrio cholera, } \\
P . \text { aeruginosa, } \\
\text { V. aglinolyticus }\end{array}$ & No activity & {$[15]$} \\
\hline
\end{tabular}

In addition to antibacterial activity, mushrooms also can cure several infectious diseases caused by viruses. Zhang et al. isolated a novel ribonuclease from fruiting bodies of $R$. formosa and checked its ability to inhibit the HIV-1 reverse transcriptase enzyme [36]. Results showed that the ribonuclease exhibited $93 \%$ inhibition at the maximum concentration tested $(30 \mu \mathrm{M})$ with an IC 50 value of $3 \mu \mathrm{M}$. The enzyme possessed certain novel features, including its unique $\mathrm{N}$-terminal sequences, temperature-resistance, and optimum acidic $\mathrm{pH}$ value suggesting this ribonuclease might have a unique role in the prevention of HIV diseases.

\subsection{Anticancer activity.}

Cancer is ranked as the second-largest cause of death after cardiovascular diseases. Despite many treatment strategies that have come up with the advancement of science, most of them are target-unspecific and with several side effects as well as costly [37]. Therefore, there is an urgent need to develop nature-based, cost-effective anticancer agents. As such, mushrooms have been reported to show anticancer activity, and many mushrooms derived compounds have already been tested for clinical trials [38-41].

In 1982, Yoo et al. tested the antitumor activity of a protein-bound polysaccharide fraction of $R$. formosa and in sarcoma 180 implanted mice[42]. It was found to inhibit $66 \%$ of a tumor at the dosage of $50 \mathrm{mg} / \mathrm{kg} / \mathrm{day}$. The tumor in two out of eight mice wholly degenerated. Alcohol extract and acid hydrolysates of $R$. botrytis displayed an antiproliferative effect on the HeLa cell line [43]. Further, Kim et al. investigated the cytotoxic effects of water, methanol, and ethyl acetate extract of the same mushroom against human colon adenocarcinoma (HT-29) and hepatocarcinoma cell (HepG2) [44]. It was found that HT-29 cells were susceptible to methanol and ethyl acetate extract. In contrast, moderate activity was shown by water extract. Recently, a novel ubiquitin-like protein, RBUP was isolated from $R$. botrytis, which exhibited apoptosis-mediated cell death in lung cancer cell lines (A549) [45]. The ethanolic extract of $R$. flava was able to inhibit the proliferation of the human breast cancer cell line (MDA-MB-231), and ergosterol peroxide was recommended to be responsible for the anticancer properties of this mushroom [19]. Likewise, methanolic and aqueous extracts of $R$. flava also showed potent antiproliferative activity against the human liver carcinoma HepG2 cell line, which could be due to the presence of gallic acid and p-coumaric acid as suggested by the authors [46]. Water extracts of fermented mycelia and culture supernatant of $R$. botryoides also showed antitumor activity against the Human HCC cell line (SMMC7721) with an $\mathrm{IC}_{50}$ value of 0.284 and 0.593 $\mathrm{mg} / \mathrm{ml}$, respectively [47]. Recently, a novel polysaccharide (RF1) was isolated from an edible species of Ramaria; R. flaccida (Fr.) Quél. and found to inhibit mouse sarcoma S180 tumors 
growth in vivo mice model. The administration of $20 \mathrm{mg} / \mathrm{kg}$ polysaccharide dosage inhibited the tumor growth rate to $48.4 \%$ and triggered Wnt and MAPK signaling pathways followed by the downregulation of interleukin levels [48].

\section{Other bioactivities of Ramaria species}

Only a few numbers of other beneficial properties have been determined in Ramaria species. Kim et al. evaluated the hepatoprotective activity of methanol extract of $R$. botrytis against liver toxicity in mice induced by benzo $(\alpha)$ pyrene [26]. Results revealed that the extract reduced the increased enzyme activities such as glutathione S-transferase and rglutamylcysteine synthetase due to the induction of benzo $(\alpha)$ pyrene. The low EC50 value $(0.109$ $\mathrm{mg} / \mathrm{ml}$ ) for DPPH radical scavenging assay also pointed towards the high antioxidant potential of the extract, which might have contributed to the hepatoprotective property of this mushroom.

A pure water-soluble glucan was obtained from fresh fruiting bodies of $R$. botrytis collected from hilly areas of Darjeeling, which consisted of $(1 \rightarrow 6)$-linked- $\beta$-D glucopyranosyl residues with the branching of $(1 \rightarrow 3)$-linked- $\beta$-D-glucopyranosyl at $O-3$ position demonstrated excellent immunostimulatory potential in a murine macrophage cell line (RAW264.7 cell) as well as in splenocyte and thymocyte cells. The glucan was found to enhance nitric oxide level and stimulate splenocyte and thymocyte proliferation rates. Hence this glucan can be developed into a potent immunostimulatory [49].

Two novel sesquiterpene derivatives named ramarin A and B, along with three known compounds, were purified from methanol extract of $R$. Formosa and represent one of the very rare mushrooms derived inhibitor of human neutrophil elastase, which can be utilized for the treatment of skin aging [50].

Öztürk et al. studied the effect of baking on the bioactivity of $R$. flava collected from a local market in Muğla-Turkey and reported that the baking process influenced higher nutrient contents and bioactivities than unbaked ones. Both baked and unbaked extracts, namely hexane and methanolic fraction, showed potential in vitro acetylcholinesterase (AChE) and butyrylcholinesterase (BChE) inhibitory activities. However, the efficacy should be evaluated with purified components and in an in vivo experimental system [51].

Recently. Bhanja et al. prepared bio metallic composite nanoparticles from $R$. botrytis polysaccharide. They tested for its antioxidant potential against different highly reactive free radicals as well as antibacterial activity towards Pseudomonas aeruginosa. The bio-nano particles showed excellent radical scavenging efficacy towards DPPH radical, nitric oxide radical, and hydrogen peroxide. It also catalyzed the reduction of p-nitrophenol, thus indicating a new direction in the field of nanotechnology-mediated biomedicine [52].

\section{Conclusion}

The updated knowledge about Ramaria sp. studied to date made available in this comprehensive review shows that coral mushroom harbors the potential as a functional therapeutic food. However still, it requires more advanced approaches for profound exploration. Most of the research works had focussed on different extracts of species. Only a few species such as $R$. formosa, $R$. botrytis, $R$. cystidiphora, and $R$. madagascariensis have been studied to identify novel compounds from their fruiting bodies. Therefore the further investigation is needed to find out their activities and mechanisms of action. The nutritional values of the genus Ramaria studied so far are entirely satisfactory. However, there is limited 
information regarding their bioavailability, mineral, and vitamin compositions. Therefore, this review recommends further exploration of this group in nutrition and mycomedicine for its upgradation from diet food to holistic mycomedicine.

\section{Funding}

This research received no external funding.

\section{Acknowledgments}

This research has no acknowledgment.

\section{Conflicts of Interest}

The authors declare no conflict of interest.

\section{References}

1. Oyetayo, O.V. Medicinal uses of mushrooms in Nigeria: Towards full and sustainable exploitation. Afr. J. Tradit. Complement. Altern. Med. 2011, 8, 267-274, https://doi.org/10.4314/ajtcam.v8i3.65289

2. Khan, M.A.; Tania, M. Nutritional and medicinal importance of Pleurotus mushrooms: An Overview. Food Rev. Int. 2011, 28, 313-329, https://doi.org/10.1080/87559129.2011.637267

3. Kanchi, S.; Inamuddin; Khan, A. Biogenic synthesis of selenium nanoparticles with edible mushroom extract: Evaluation of cytotoxicity on prostate cancer cell lines and their antioxidant, and antibacterial activity. Biointerface Res. Appl. Chem. 2020, 10, 6629-6639, https://doi.org/10.33263/BRIAC106.66296639.

4. Sinha, S.K.; Upadhyay, T.K.; Sharma, S.K. Nutritional-medicinal profile and quality categorization of fresh white button mushroom. Biointerface Res. Appl. Chem. 2021, 11, 8669-8685, https://doi.org/10.33263/BRIAC112.86698685.

5. Chakraborty, N.; Banerjee, A.; Sarkar, A.; Ghosh, S.; Acharya, K. Mushroom polysaccharides: A potent immune-modulator. Biointerface Res. Appl. Chem. 2021, 11, 8915-8930.

6. Coker, W.C. The Clavarias of the United States and Canada. Chapel Hill, N.C. 1923; pp. 209.

7. Marr, C.D.; Stuntz, D.E. Ramaria of Western Washington. Biblioth Mycol. 1973, 38, 1-232.

8. Dattaraj, H.R.; Sridhar, K.R.; Jagadish, B.R.; Pavithra, M. Bioactive potential of the wild edible mushroom Ramaria versatilis. Studies in Fungi 2020, 5, 73-83, https://doi.org/10.5943/sif/5/1/7.

9. González-Ávila, P.A.; Luna-Vega, I.; Rios, M.V.; Lira, R.; Cifuentes, J. Current knowledge and importance of the order Gomphales (Fungi: Basidiomycota) in Mexico. Nova Hedwigia 2013, 97, 55-86, https://doi.org/10.1127/0029-5035/2013/0099.

10. Thu, Z.M.; Myo, K.K.; Aung, H.T.; Clericuzio, M.; Armijos, C.; Vidari, G. Bioactive phytochemical constituents of wild edible mushrooms from Southeast Asia. Molecules 2020, 25, https://doi.org/10.3390/molecules25081972.

11. Debnath, S.; Debnath, B.; Das, P.; Ajay, K.S. Review on an ethnomedicinal practices of wild mushrooms by the local tribes of India. J Appl Pharm 2019, 9, 144-156, https://doi.org/10.7324/JAPS.2019.90818.

12. Barros, L.; Venturini, B.A.; Baptista, P.; Estevinho, L.M.; Ferreira, I.C. Chemical composition and biological properties of portuguese wild mushrooms: A Comprehensive Study. J Agric Food Chem. 2008, 28, 3856-62, https://doi.org/10.1021/jf8003114.

13. Colak, A.; Faiz, O.; Sesli, E. Nutritional composition of some wild edible mushrooms. Turk. J. Biochem. 2009, 34, 25-31.

14. Dundar, A.; Hilal, A.; Yildiz, A. Yield performance and nutritional contents of three oyster mushroom species cultivated on wheat stalk. Afr J Biotechnol. 2008, 7, 3497-3501.

15. Sharma, S.K.; Gautam, N. Chemical and bioactive profiling, and biological activities of coral fungi from Northwestern Himalayas. Sci. Rep. 2017, 7, https://doi.org/10.1038/srep46570.

16. De Silva, D.D.; Rapior, S.; Hyde, K.D.; Bahka, A.H. Medicinal mushrooms in prevention and control of diabetes mellitus. Fungal Divers. 2012, 56, 1-29, https://doi.org/10.1007/s13225-012-0187-4.

17. Gursoy, N.; Sarikurkcu, C.; Tepe, B.; Solak, M.H. Evaluation of antioxidant activities of 3 edible mushrooms: Ramaria flava (Schaef.: Fr.) Quél., Rhizopogon roseolus (Corda) T.M. Fries., and Russula delica Fr. Food Sci. Biotechnol. 2010, 19, 691-696, https://doi.org/10.1007/s10068-010-0097-8.

18. Gezer, K.; Duru, M.E.; Kivrak, I.; Turkoglu, A.; Mercan, N.; Turkoglu, H.; Gulcan, S. Free-radical scavenging capacity and antimicrobial activity of wild edible mushroom from Turkey. Afr. J. Biotechnol. 2006, 5, 1924-1928. 
19. Liu, K.; Wang, J.; Zhao, L.; Wang, Q. Anticancer, antioxidant and antibiotic activities of mushroom Ramaria flava. Food Chem. Toxicol. 2013, 58, 375-380, https://doi.org/10.1016/j.fct.2013.05.001.

20. Aprotosoaie, A.C.; Zavastin, D.E.; Mihai, C.T.; Voichita, G.; Gherghel, D.; Silion, M.; Trifan, A.; Miron, A. Antioxidant and antigenotoxic Potential of Ramaria largentii Marr \& D.E. Stuntz, A wild edible mushroom collected from Northeast Romania. Food Chem. Toxicol. 2017, 108, 429-437, https://doi.org/10.1016/j.fct.2017.02.006.

21. Toledo, C.V.; Barroetaveña, C.; Fernandes, Â.; Barros, L.; Ferreira, I.C.F.R. Chemical and antioxidant properties of wild edible mushrooms from native Nothofagus spp. Forest, Argentina. Molecules 2016, 21, https://doi.org/10.3390/molecules21091201.

22. Ramesh, C.H.; Pattar, M.G. Antimicrobial properties, antioxidant activity and bioactive compounds from six wild edible mushrooms of Western Ghats of Karnataka, India. Pharmacogn. Res. 2010, 2, 107-112, https://doi.org/10.4103/0974-8490.62953.

23. Acharya, K.; Das, K.; Paloi, S.; Dutta, A.K.; Hembrom, M.E.; Khatua, S.; Parihar, A. Exploring a novel edible mushroom Ramaria subalpina: Chemical characterization and antioxidant activity. Pharmacogn. J. 2017, 9, 30-34, https://doi.org/10.5530/pj.2017.1.6.

24. Khatua, S.; Mitra, P.; Chandra, S.; Acharya, K. In vitro protective ability of Ramaria aurea against free radical and identification of main phenolic acids by HPLC. J Herbs Spices Med Plants 2015, 21, 380-391, https://doi.org/10.1080/10496475.2014.994085.

25. Han, S.R.; Kim, K.H.; Kim, H.J.; Jeong, S.H.; Oh, T.J. Comparison of biological activities using several solvent extracts from Ramaria botrytis. Indian J. Sci. Technol. 2017, 9, https://doi.org/10.17485/ijst/2016/v9i41/103921.

26. Kim, H.J.; Lee, K.R. Effect of Ramaria botrytis Methanol extract on antioxidant enzyme activities in Benzo (a) Pyrene-treated mice. Korean J. Food Sci. Technol. 2003, 35, 286-90, https://doi.org/10.4489/KJM.2003.31.1.034.

27. Alves, M.J.; Ferreira, I.C.; Dias, J.; Teixeira, V.; Martins, A.; Pintado, M. A review on antimicrobial activity of mushroom (Basidiomycetes) extracts and isolated compounds. Planta Med. 2012, 78, 1707-18, https://doi.org/10.1055/s-0032-1315370.

28. Gebreyohannes, G.; Nyerere, A.; Bii, C.; Sbhatu, D.B. Determination of antimicrobial activity of extracts of indigenous wild mushrooms against pathogenic organisms. Evid Based Complement Alternat Med 2019, 7, https://doi.org/10.1155/2019/6212673.

29. Román, P.G.R.; Mantilla, N.B.; Flórez, S.A.C.; Mandal, S.D.; Passari, K.A.; Ruiz-Villáfan, B.; RodríguezSanoja, R.; Sánchez, S. Antimicrobial and antioxidant potential of wild edible mushrooms. An Introduction to Mushroom. 2020; https://doi.org/10.5772/intechopen.90945.

30. Dasgupta, A.; Acharya, K. Mushrooms: An emerging resource for therapeutic terpenoids. 3 Biotech 2019, 9, https://doi.org/10.1007/s13205-019-1906-2.

31. Bala, N.; Aitken, E.A.B.; Fechner, N.; Cusack, A.; Steadman, K.J. Evaluation of antibacterial activity of Australian basidiomycetous macrofungi using a high-throughput 96-well plate assay. Pharm. Biol. 2011, 49, 492-500, https://doi.org/10.3109/13880209.2010.526616.

32. Rai, M.; Sen, S.; Acharya, K. Antimicrobial activity of four wild edible mushrooms from Darjeeling Hills, West Bengal, India. Int. J. Pharmtech. Res. 2013, 5, 949-956.

33. Bala, N.; Aitken, E.A.B.; Cusack, A.; Steadman, K. Antimicrobial potential of Australian macrofungi extracts against foodborne and other pathogens. Phytother Res. 2011, 26, 465-9, https://doi.org/10.3109/10.1002/ptr.3563.

34. Centko, R.M.; Ramón-García, S.; Taylor, T.; Patrick, B.O.; Thompson, C.J.; Miao, V.P.; Andersen, R.J.; Ramariolides, A.-D. Antimycobacterial Butenolides Isolated from the Mushroom Ramaria cystidiophora. J Nat Prod. 2012, 75, 2178-82, https://doi.org/10.1021/np3006277.

35. Lehmann, J.; Richers, J.; Pöthig, A.; Sieber, S. A. Synthesis of Ramariolide natural products and discovery of their targets in Mycobacteria. Chem Commun. 2017, 53, 107-110, https://doi.org/10.1039/C6CC08365J.

36. Zhang, R.; Tian, G.; Zhao, Y.; Zhao, L.; Wang, H.; Gong, Z.; Ng, T.B.A. Novel Ribonuclease with HIV-1 reverse transcriptase inhibitory activity purified from the fungus Ramaria formosa. J. Basic Microbiol. 2015, 55, 269-275.

37. Baldo, B.A.; Pham, N.H. Adverse reactions to targeted and non-targeted chemotherapeutic drugs with emphasis on hypersensitivity responses and the invasive metastatic switch. Cancer Metastasis Rev. 2013, 32, 723-61, https://doi.org/10.1007/s10555-013-9447-3.

38. Joseph, T.P.; Chanda, W.; Padhiar, A.A.; Batool, S.; LiQun, S.; Zhong, M.; Huang, M. A Preclinical Evaluation of the Antitumor Activities of Edible and Medicinal Mushrooms: A Molecular Insight. Integrative Cancer Therapies 2017, 17, 200-209, https://doi.org/10.1177/1534735417736861.

39. Chaitanya, M.V.N.L.; Jose, A.; Ramalingam, P.; Mandal, S.C.; Kumar, P.N. Multi-targeting cytotoxic drug leads from mushrooms. Asian Pac J Trop Med 2019, 12, 531-536, https://doi.org/10.4103/19957645.272482 .

40. Badalyan, S.M.; Barkhudaryan, A.; Rapior, S. Recent progress in research on the pharmacological potential of mushrooms and prospects for their clinical application. In: Med Mushrooms. Agrawal, D.; Dhanasekaran, M. (eds) Springer, Singapore. 2019; https://doi.org/10.1007/978-981-13-6382-5_1. 
41. Nandi, S.; Sikder, R.; Acharya, K. Secondary Metabolites of Mushrooms: A potential source for anticancer therapeutics with translational opportunities. In: Advancing Frontiers in Mycology \&Mycotechnology. Satyanarayana, T.; Deshmukh, S.; Deshpande, M. (eds) Springer, Singapore, 2019; https://doi.org/10.1007/978-981-13-9349-5_23.

42. Yoo, I.S.; Woo, M.S.; Choi, E.C.; Kim, B.K. Studies on constituents of higher fungi of Korea (XXXIX) antitumor components of Ramaria formosa. The Korean J Mycol. 1982, 10, 165-171.

43. Chung, K.S. The effects of mushroom components on the proliferation of HeLa cell line in Vitro. Arch. Pharmacal. Res. 1979, 2, 25-33, https://doi.org/10.1007/BF02856430

44. Kim, H.J.; Lee, I.S.; Lee, K.R. Antimutagenic and anticancer effects of Ramaria botrytis (Fr.) Rick extracts. J Korean Soc. Food Sci. Nutr. 1999, 28, 1321-5, https://doi.org/10.3746/jkfn.2007.36.11.1371.

45. Zhou, R.; Han, Y.-J.; Zhang, M.-H.; Zhang, K.-R.; Ng, T.B.; Liu, F. Purification and characterization of a novel ubiquitin-like antitumour protein with hemagglutinating and deoxyribonuclease activities from the edible mushroom Ramaria botrytis. AMB Expr. 2017, 7, https://doi.org/10.1186/s13568-017-0346-9.

46. Sadi, G.; Kaya, A.; Yalcin, H.A.; Emsen, B.; Kartal, D.İ.; Altay, A. Wild edible mushrooms from turkey as possible anticancer agents on HepG2 cells together with their antioxidant and antimicrobial properties. Int. J. Med. Mushrooms. 2016, 18, 83-95, https://doi.org/10.1615/IntJMedMushrooms.v18.i1.100.

47. Gao, X.J.; Yan, P.S.; Wang, J.B.; Yu, J.J. ACE inhibitory, antitumor and antioxidant activities of submerged culture materials of three medicinal mushrooms. App. Mech. Mater. 2012, 145, 179-183, https://doi.org/10.4028/www.scientific.net/AMM.145.179.

48. Dong, M.; Hou, Y.; Ding, X. Structure identification, antitumor activity and mechanisms of a novel polysaccharide from Ramaria flaccida (Fr.) Quél. Oncol. Lett 2020, 20, 2169-2182, https://doi.org/10.3892/ol.2020.11761.

49. Bhanja, S.K.; Rout, D.; Patra, P.; Nandan, C.K.; Behera, B.; Maiti, T.K.; Islam, S.S. Structural studies of an immunoenhancing glucan of an ectomycorrhizal fungus Ramaria botrytis. Carbohydr. Res. 2014, 374, 5966, https://doi.org/10.1016/j.carres.2013.03.023.

50. Kim, K.-C.; Kwon, Y.-B.; Jang, H.-D.; Kim, J.W.; Jeong, J.C.; Lee, I.-S.; Ha, B.-J.; Yoo, I.-D. Study on the antioxidant and human neutrophil elastase inhibitory activities of mushroom Ramaria formosa extracts. $J$. Soc. Cosmet. Sci. Korea 2016, 42, 269-278, https://doi.org/10.15230/SCSK.2016.42.3.269.

51. Öztürk, M.; Tel, G.; Öztürk, F.A.; Duru, M.E. The cooking effect on two edible mushrooms in Anatolia: Fatty acid composition, total bioactive compounds, antioxidant and anticholinesterase activities. Rec. Nat. Prod. 2013, 8, 189-194.

52. Bhanja, S.K.; Samanta, S.K.; Mondal, B.; Jana, S.; Ray, J.; Pandey, A.; Tripathy, T. Green synthesis of $\mathrm{Ag} @ \mathrm{Au}$ bimetallic composite nanoparticles using a polysaccharide extracted from Ramaria botrytis mushroomand performance in catalytic reduction of 4-nitrophenol and antioxidant, antibacterial activity, Environ. Nanotechnol. Monit. Manag. 2020, 14, https://doi.org/10.1016/j.enmm.2020.100341.

53. Ouzouni, P.K.; Petridis, D.; Koller, W.D.; Riganakos, K.A. Nutritional value and metal content of wild edible mushrooms collected from West Macedonia and Epirus, Greece. Food Chem. 2009, 115, 575-1580, https://doi.org/10.1016/j.foodchem.2009.02.014.

54. Agrahar-Murugkar, D.; Subbulakshmi, G. Nutritional value of edible wild mushrooms collected from the Khasi Hills of Meghalaya. Food Chem. 2005, 89, 599-603. https://doi.org/10.1016/j.foodchem.2004.03.042

55. Li, H. Extraction, purification, characterization and antioxidant activities of polysaccharides from Ramaria botrytis (Pers.) Ricken. L. Chem. Cent. J. 2017, 11, https://doi.org/10.1186/s13065-017-0252-x.

56. Rai, M.; Acharya, K. Proximate composition, free radical scavenging and NOS activation properties of Ramaria aurea. Research J. Pharm. Tech. 2012, 5, 1421-1427.

57. An, G.-H.; Han, J.-G.; Cho, J.-H. Comparison of the antioxidant activity and nutritional contents of ectomycorrhizal mushroom extracts in Korea. J Mushrooms 2020, 18, 164-173, https://doi.org/10.14480/JM.2020.18.2.164.

58. Krupodorova, T.; Sevindik, M. Antioxidant potential and some mineral contents of wild edible mushroom Ramaria stricta. AgroLife Sci. J. 2020, 9, 186-191.

59. Pala, S.A.; Wani, A.H.; Ganai, B.A. Antimicrobial potential of some wild Macromycetes collected from Kashmir Himalayas. Plant Science Today 2019, 6, 137-146, https://doi.org/10.14719/pst.2019.6.2.503.

60. Giri, S.; Biswas, G.; Pradhan, P.; Mandal, S.C.; Acharya, K. Antimicrobial activities of basidiocarps of wild edible mushrooms of West Bengal, India. Int. J. Pharmtech. Res. 2012, 4, 1554-60. 